\title{
Functional architecture of the retromer cargo-recognition complex
}

\author{
Aitor Hierro ${ }^{1,2}$, Adriana L. Rojas ${ }^{1,2}$, Raul Rojas $^{3}$, Namita Murthy $^{3}$, Grégory Effantin ${ }^{4}$, Andrey \\ V. Kajava ${ }^{5}$, Alasdair C. Steven ${ }^{4}$, Juan S. Bonifacino ${ }^{3}$, and James H. Hurley ${ }^{1,6}$ \\ 1 Laboratory of Molecular Biology, National Institute of Diabetes and Digestive and Kidney Diseases, \\ National Institutes of Health, U. S. Department of Health and Human Services, Bethesda, MD 20892, USA \\ 3 Cell Biology and Metabolism Branch, National Institute of Child Health and Human Development, National \\ Institutes of Health, U. S. Department of Health and Human Services, Bethesda, MD 20892, USA \\ 4Laboratory of Structural Biology, National Institute of Arthritis, Musculoskeletal, and Skin Diseases, \\ National Institutes of Health, U. S. Department of Health and Human Services, Bethesda, MD 20892, USA \\ 5 Centre de Recherches de Biochimie Macromoléculaire, CNRS, University of Montpellier, 1919 Route de \\ Mende, 34293 Montpellier, France.
}

\section{Abstract}

The retromer complex 1,2 is required for the sorting of acid hydrolases to lysosomes 3-7, transcytosis of the polymeric Ig receptor 8 , Wnt gradient formation 9,10, iron transporter recycling 11, and processing of the amyloid precursor protein 12 . Human retromer consists of two smaller complexes, the cargo recognition Vps26:Vps29:Vps35 heterotrimer, and a membrane-targeting heterodimer or homodimer of SNX1 and/or SNX2 13. The crystal structure of a Vps29:Vps35 subcomplex shows how the metallophosphoesterase-fold subunit Vps29 14,15 acts as a scaffold for the C-terminal half of Vps35. Vps35 forms a horseshoe-shaped right-handed $\alpha$-helical solenoid whose concave face completely covers the metal-binding site of Vps29 and whose convex face exposes a series of hydrophobic interhelical grooves. Electron microscopy shows that the intact Vps26:Vps29:Vps35 complex is a stick-shaped, somewhat flexible, structure, $\sim 21 \mathrm{~nm}$ long. A hybrid structural model derived from crystal structures, electron microscopy, interaction studies, and bioinformatics shows that the $\alpha$-solenoid fold extends the full length of Vps35, and that Vps26 is bound at the opposite end from Vps29. This extended structure presents multiple binding sites for the SNX complex and receptor cargo, and appears capable of flexing to conform to curved vesicular membranes.

The retromer cargo recognition complex consists of the 38-kDa Vps26, 20-kDa Vps29, and 92-kDa Vps35 subunits. The structures of the two smaller subunits have been determined in isolation. Vps26 is a structural cousin of the arrestins 16, a family of trafficking proteins that directly bind to cell surface receptors and direct their internalization. Vps29 has a metallophosphoesterase fold 14,15 that can bind two metal ions. Compared to functional metallophosphoesterases, a key His that serves as a catalytic base in the

\footnotetext{
6To whom correspondence should be addressed:James H. Hurley, (301) 402-4703, fax (301) 480-0639; E-mail: hurley@ helix.nih.gov.

2 These authors contributed equally.

Author contributions A. L. R. and A. H. expressed and purified protein complexes, crystallized the Vps29:Vps35 C-terminal

subcomplex, collected crystallographic data, and determined and refined the crystal structure; A. H. carried out phosphatase assays; R. R. and N. M. carried out immunoprecipitation and optical microscopy studies; G. E. and A. C. S. carried out and interpreted electron microscopy studies; A. V. K. carried out sequence analysis; and J. H. H., J. S. B., and A. C. S. designed the study. A. H. and A. L. R. contributed equally to this study.

Author information The authors declare that they have no competing financial interests. Correspondence and requests for materials should be addressed to J.H.H. (hurley@ helix.nih.gov). Crystallographic coordinates have been deposited with the Protein Data Bank with accession number $2 \mathrm{R} 17$.
} 
metallophosphoesterase active site is replaced by Phe 63 . Thus Vps29 is completely inactive with respect to generic phosphatase substrates 15 . However, metal-dependent activity in vitro against a phosphorylated peptide from a major retromer cargo, the cation-independent mannose 6-phosphate receptor (CI-MPR), has been reported 17. Despite its centrality to multiple trafficking pathways, the precise function of retromer has been enigmatic. Various proposals have emphasized potential roles as a coat, adaptor, or cargo protein phosphatase. Here we take a structural approach to gain insight into the function of retromer.

The crystal structure of a Vps29:Vps35 subcomplex, containing the C-terminal 40\% of the large Vps35 subunit, was determined at $2.8 \AA$ resolution (Fig. 1a, Fig. S1, Table S1). The Cterminal portion of Vps35 consists of a single right-handed superhelix with a pitch of $12 \AA$ and a total of 13 helices (Fig. 1a, S2). Vps35 resembles many other helical solenoid proteins, including other important players in coated vesicle trafficking. These include the trunk domains of the large subunits of the AP adaptor protein complexes 18,19 (Fig. S3), whose function is to link clathrin to cargo and membranes, the helical repeat regions of the heavy chain of clathrin itself 20,21 , and the Sec13/Sec31 assembly unit of the COPII coat 22 . Vps35 wraps itself nearly halfway around the Vps29 subunit, burying $3300 \AA 2$ of solvent-accessible surface area (Fig. $1 b, c)$. The Vps35 binding site on Vps29 includes the entire metal-binding site, as well as flanking residues. Vps29 Ile-91, previously shown to contact Vps3515, interacts extensively with Vps35 near the center of the Vps29:Vps35 interface (Fig. 1a). Vps35 and its C-terminal fragment are both poorly stable when expressed in isolation, consistent with a fold that depends on scaffolding by Vps 29 for its stability. The even-numbered helices of the convex face of Vps35 are splayed apart from each other because the solenoid is curved. This surface thus consists of a series of ridges separating hydrophobic grooves, suggestive of potential cargo binding sites (Fig. 1d). These grooves are reminiscent of the CI-MPR binding site on the helical solenoidal VHS domains of the GGA clathrin adaptor proteins 23,24 . The binding site for Vps10 to yeast Vps35 25 maps to part of this surface (Fig. 1d).

Two models have been proposed for the function of Vps29. In the first, Vps29 is a scaffold for interaction with Vps35 and SNX115 with no phosphatase activity of functional significance. In the second, Vps29 is an active protein phosphatase for the CI-MPR and perhaps other receptors 17. The complete burial of the putative Vps29 metallophosphoesterase active site prompted us to revisit the report of CI-MPR dephosphorylation activity by retromer 17 . Working with preparations purified to homogeneity, neither the complete Vps26:Vps29:Vps35 complex nor the Vps29:Vps35 subcomplex had detectable activity (Fig. S4). Furthermore, Vps29 alone had no detectable activity.

The lack of in vitro phosphatase activity suggests, but does not prove, that the main function for the metallophosphoesterase site is to scaffold Vps35. Vps29 must associate with Vps35 to maintain its known biological functions in sorting 15 . We considered whether a conformational change in Vps35 could expose the Vps29 metallophosphoesterase site without completely disassembling the complex. One region of Vps35 covers the putative Vps29 active site and is thus potentially an inhibitory contact site, while another region is remote from the putative active site and unlikely to be involved in inhibition (Fig. 1b,c). Each of these two regions comprises roughly half of the total contact area. The scaffold model predicts that mutations that selectively disrupt the putative inhibitory region should prevent the assembly of the complex. The phosphatase model, on the other hand, predicts that these mutations should allow integration into the retromer but lead to constitutive exposure of the metallophosphoesterase site. HeLa cells were transfected with mammalian expression plasmids encoding HA-tagged wild-type or mutant Vps35 constructs carrying multiple substitutions on residues (Fig. 1b, gray residues) covering the putative Vps29-phosphatase active site or residues (Fig. 1b, slate residues) distal to this region. After transfection, the proteins were isolated by immunoprecipitation and analyzed by SDS- PAGE and immunoblotting with antibodies to 
Vps26 and Vps29 (Fig. 2a, top). We found that wild-type HA-Vps35 can assemble with endogenous Vps29 and Vps26. In contrast, all of the HA-tagged Vps35 mutants in both regions prevented assembly with Vps29, consistent with the scaffolding model. All of the recombinant HA-tagged Vps35 constructs expressed at similar levels in the transfected cells (Fig 2a, bottom). In addition, these HA-tagged Vps35 mutants all assembled with Vps26 (Fig. 2a), indicating they are capable of proper folding and that the Vps26 and Vps29 binding sites are independent of one another. We used double-labeling fluorescent microscopy to study the cellular distribution of these HA-tagged constructs (Fig. S5a). Unlike wild-type HA-Vps35, which is mostly endosomal, all of the mutant HA-Vps35 constructs showed a diffuse cytosolic distribution.

Similarly, we transfected wild-type myc-tagged Vps29 and versions harboring mutations of Vps35-interacting residues surrounding the metallophosphoesterase binding site (Fig. 1c, gray residues) and remote from it (Fig. 1c, slate residues). These constructs were

immunoprecipitated and analyzed by SDS-PAGE and immunoblotting using antibodies to Vps35 and Vps26 (Fig. 2b, top). All but one (L101D) of the characterized myc-tagged Vps29 mutants failed to assemble with endogenous Vps35, re-emphasizing that all regions of the interface are important for assembly. We also tested nine mutants of the Vps29

metallophosphoesterase site and found that only three (D8N, H86A and H117A) assembled partially with Vps35, while the remaining mutants did not assemble (Table S2). The levels of these mutants were much lower than wild-type myc-tagged Vps29 and almost undetectable in lysates by immunoblot analysis (Table S2). This led us to conclude that an intact metal-binding site is required for the stability of $\mathrm{Vps} 29$.

Indeed, the use of a metallophosphoesterase fold for non-catalytic purposes may be common. Of 5307 putative metallophosphoesterase catalytic domains, only $41 \%$ of the sequences conserve eight key residues directly involved in metal binding or catalysis. Even though catalytically inactivate metallophosphoesterases appear to comprise the majority of this vast protein fold family, little is known about their functions. It will be interesting to learn if the Vps29 example is a paradigm for other members of this class. How might so many such folds have evolved? The structure of full-length protein phosphatase 5 shows that its TPR domain, a helical solenoid, is inserted into and sterically blocks the active site of the catalytic domain 26 , and suggests the nature of a potential evolutionary precursor. We suggest that most of the metal ligands were retained during evolution for stability reasons, despite their lack of catalytic function.

The intact recombinant Vps26:Vps29:Vps35 complex was found to contain an equimolar ratio of the three subunits by Coomassie-stained SDS-PAGE (Fig. 2c) and by quantitative amino acid analysis (not shown). The elution of the complex on gel filtration chromatography (not shown) is potentially consistent with either an elongated 1:1:1 complex or a globular 2:2:2 complex. Given that the electron microscopy described below indicates that the structure is elongated and occupies a volume consistent with a 1:1:1 complex, we favor this interpretation. Through co-expression of truncated recombinant proteins, we mapped the interaction of Vps 26 and Vps35 to the C-terminal lobe of the former, and the N-terminal 165 amino acids 4 of the latter (Fig. 2d). The organization of the large subunit, Vps35, is central to understanding the structure of retromer. Analysis of the Vps 35 sequence showed that it consists of regular repeats of a two-helix motif (Fig. 3a), strongly suggesting that the $\alpha$-solenoid visualized in the crystallized fragment extends throughout essentially the entire structure. The total number of helices is anticipated to be 34, including the 13 in Vps35-C. This corresponds to a contour length of $\sim 204 \AA$ for the complete molecule, given a pitch of $12 \AA$ per two-helical turn of the solenoid in the crystallized portion. The conformations of other $\alpha$-solenoids vary from ringlike to quasilinear 27,28 . How is the putative $\alpha$-solenoid arranged in this case? To investigate this question, we performed negative staining electron microscopy. The images (e.g. Fig. S6) 
exhibit some variability but many molecules are filamentous and about $210 \AA$ Along. To enhance detail, we performed image averaging after alignment and classification. The class averages exhibit some variation in local structure but are rather consistent in length, between 210 and $220 \AA$ (Fig. 3b). Each exhibits five or six stain-excluding densities. These data can be accounted for by a model consisting of the Vps29:Vps35-C heterodimer at one end, extended by $\sim 135 \AA$ of $\alpha$-solenoid with Vps26 attached near its distal end (Fig. 3c, d). This model was tested by generating resolution-limited projections from the model for comparison with the negatively stained class averages (Fig. 3c). At one end, the structure is consistent with projections of the Vps29:Vps35-C subcomplex. The respective class averages correspond to different side-views of this assembly, with some flexing about its mid-section (Fig. S7). These data strongly support the inferred continuation of the Vps35 $\alpha$-solenoid in an approximately linear conformation, with Vps26 bound near its end.

The overall structural model suggests a mechanism for cooperative binding to multiple membrane-anchored molecules (Fig. 4a). The membrane-binding SNX1 subunit interacts with a groove on Vps29 (at Leu-25 and Leu-152) 15 on the face distal to Vps35, and to two different sites on the $\mathrm{N}$ - and $\mathrm{C}$-terminal halves of $\mathrm{Vps} 35$ 4. The $\mathrm{N}$ - and $\mathrm{C}$-terminal halves of yeast $\mathrm{Vps} 35$ have been shown to interact with the distinct cargoes A-ALP and Vps10, respectively 25 . The Vps35 binding site on the A-ALP cytosolic domain is within 20 residues of the A-ALP transmembrane region 29, dictating that the $\mathrm{N}$-terminal half of Vps35 must be close to the membrane. The flexibility of the cargo recognition complex suggests that it could adapt to the shape of curved tubulovesicular membranes and so bind multiple membrane-embedded cargo molecules. The curvature and contour length of the SNX dimer can be estimated from the related structure of the amphiphysin BAR domain 30, which has a contour length of $150 \AA$ along its inner membrane binding surface. The cargo recognition complex has at least three distinct points of interaction with the SNX dimer. Their contour lengths are similar, suggesting that they could form curved side-by-side arrays at a 1:1 ratio (Fig. 4b). Although speculative, this conceptual model helps lay a foundation for further analysis of the assembly of the full retromer complex on cargo-bearing vesicles.

The clathrin and COPII coats, which direct the budding of roughly spherical vesicles, have been the principal paradigms for the structural dissection of coat complexes 20-22. These two coats consist of an inner layer (the clathrin adaptors and the Sec23/Sec24 complex, respectively) that binds to cargo proteins and lipids, and an outer layer that forms an organizing cage (composed of clathrin and the Sec13/Sec31 complex, respectively) but does not directly contact cargo or lipids. Retromer has extensive structural similarities to these classical systems: Vps26 looks like the clathrin adaptor ßarrestin 16, while Vps 35 has an $\alpha$-solenoid fold similar to the AP trunk domains and to the clathrin heavy chain itself. On the other hand, retromer contrasts sharply in that the inner layer functions of the classical coats, binding to cargo and membrane lipids, are divided between the cargo recognition and SNX complexes, not shared by one inner complex. Neither the cargo recognition nor the SNX complex appears to serve as an outer coat, and it is unclear if an outer coat exists for this system. The apparent lack of an outer coat in this system is consistent with the absence of a classical electron-dense coat on vesicles of retrograde trafficking 2 . The putative single-layer "cage free" organization of the system seems ideally designed for the coating of tubular vesicles of variable length. The rough cylindrical symmetry and ill-defined endpoints present very different geometric constraints than the quasi-spherical vesicles of endocytosis and the secretory pathway, and thus demand different solutions. 


\section{METHODS SUMMARY}

The crystal structure of the Vps29:Vps35 C-terminal subcomplex was determined by single anomalous dispersion at the Se edge and refined to an R-free value of 0.278 at $2.8 \AA$ resolution. Full methods and associated references are available in the online version of the paper.

\section{Acknowledgements}

We thank H. Shi for contributing to the early stages of this project, X. Zhu and H.T. Tsai for technical assistance, B. Canagarajah for bioinformatics and computational crystallography support, E. Tyler for artwork, Z.-Q. Fu and all the staff of APS SER-CAT beamline 22 for assistance with data collection, and C. Haft for antibodies to retromer. Use of the APS was supported by the U. S. DOE, Basic Energy Sciences, Office of Science, under Contract No.W-31-109Eng-38. This project was funded by the Intramural Programs of NIDDK, NICHD, and NIAMS, NIH.

\section{References}

1. Seaman MN. Recycle your receptors with retromer. Trends Cell Biol 2005;15:68-75. [PubMed: 15695093]

2. Bonifacino JS, Rojas R. Retrograde transport from endosomes to the trans-Golgi network. Nat. Rev. Mol. Cell Biol 2006;7:568-579. [PubMed: 16936697]

3. Seaman MNJ, McCaffery JM, Emr SD. A membrane coat complex essential for endosome-to-Golgi retrograde transport in yeast. J. Cell Biol 1998;142:665-681. [PubMed: 9700157]

4. Haft CR, et al. Human orthologs of yeast vacuolar protein sorting proteins Vps26, 29, and 35: Assembly into multimeric complexes. Mol. Biol. Cell 2000;11:4105-4116. [PubMed: 11102511]

5. Arighi CN, Hartnell LM, Aguilar RC, Haft CR, Bonifacino JS. Role of the mammalian retromer in sorting of the cation-independent mannose 6-phosphate receptor. J Cell Biol 2004;165:123-33. [PubMed: 15078903]

6. Carlton J, et al. Sorting nexin-1 mediates tubular endosome-to-TGN transport through coincidence sensing of high-curvature membranes and 3-phosphoinositides. Curr. Biol 2004;14:1791-1800. [PubMed: 15498486]

7. Seaman MN. Cargo-selective endosomal sorting for retrieval to the Golgi requires retromer. J Cell Biol 2004;165:111-22. [PubMed: 15078902]

8. Verges M, et al. The mammalian retromer regulates transcytosis of the polymeric immunoglobulin receptor. Nat. Cell Biol 2004;6:763-769. [PubMed: 15247922]

9. Coudreuse DYM, Roel G, Betist MC, Destree O, Korswagen HC. Wnt gradient formation requires retromer function in Wnt-producing cells. Science 2006;312:921-924. [PubMed: 16645052]

10. Prasad BC, Clark SG. Wnt signaling establishes anteroposterior neuronal polarity and requires retromer in C. elegans. Development 2006;133:1757-1766. [PubMed: 16571624]

11. Strochlic TI, Setty TG, Sitaram A, Burd CG. Grd19/Snx3p functions as a cargo-specic adapter for retromer-dependent endocytic recycling. J. Cell Biol 2007;177:115-125. [PubMed: 17420293]

12. Small SA, et al. Model-guided microarray implicates the retromer complex in Alzheimer's disease. Annals Of Neurology 2005;58:909-919. [PubMed: 16315276]

13. Rojas R, Kametaka S, Haft CR, Bonifacino JS. Interchangeable but essential functions of SNX1 and SNX2 in the association of retromer with endosomes and the trafficking of the mannose 6-phosphate receptors. Mol Cell Biol 2007;27:1112-1124. [PubMed: 17101778]

14. Wang D, et al. Crystal Structure of Human Vacuolar Protein Sorting Protein 29 Reveals a Phosphodiesterase/Nuclease-like Fold and Two Protein-Protein Interaction Sites. J Biol Chem 2005;280:22962-7. [PubMed: 15788412]

15. Collins BM, Skinner CF, Watson PJ, Seaman MN, Owen DJ. Vps29 has a phosphoesterase fold that acts as a protein interaction scaffold for retromer assembly. Nat Struct Mol Biol. 2005

16. Shi H, Rojas R, Bonifacino JS, Hurley JH. The retromer subunit Vps26 has an arrestin fold and binds Vps35 through its C-terminal domain. Nature Structural \& Molecular Biology 2006;13:540-548.

17. Damen E, Krieger E, Nielsen JE, Eygensteyn J, van Leeuwen JEM. The human Vps29 retromer component is a metallo-phosphoesterase for a cation-independent mannose 6-phosphate receptor substrate peptide. Biochem. J 2006;398:399-409. [PubMed: 16737443] 
18. Collins BM, McCoy AJ, Kent HM, Evans PR, Owen DJ. Molecular architecture and functional model of the endocytic AP2 complex. Cell 2002;109:523-535. [PubMed: 12086608]

19. Heldwein EE, et al. Crystal structure of the clathrin adaptor protein 1 core. Proc. Natl. Acad. Sci. U. S. A 2004;101:14108-14113. [PubMed: 15377783]

20. Ybe JA, et al. Clathrin self-assembly is mediated by a tandemly repeated superhelix. Nature 1999;399:371-375. [PubMed: 10360576]

21. Fotin A, et al. Molecular model for a complete clathrin lattice from electron cryomicroscopy. Nature 2004;432:573-579. [PubMed: 15502812]

22. Fath S, Mancias JD, Bi X, Goldberg J. Structure and organization of coat proteins in the COPII cage. Cell 2007;129:1251-1252. [PubMed: 17604713]

23. Misra S, Puertollano R, Kato Y, Bonifacino JS, Hurley JH. Structural basis for acidic-cluster-dileucine sorting-signal recognition by VHS domains. Nature 2002;415:933-937. [PubMed: 11859375]

24. Shiba T, et al. Structural basis for recognition of acidic-cluster dileucine sequence by GGA1. Nature 2002;415:937-941. [PubMed: 11859376]

25. Nothwehr SF, Bruinsma P, Strawn LA. Distinct domains within Vps35p mediate the retrieval of two different cargo proteins from the yeast prevacuolar/endosomal compartment. Mol. Biol. Cell 1999;10:875-890. [PubMed: 10198044]

26. Yang J, et al. Molecular Basis for TPR Domain-Mediated Regulation of Protein Phosphatase 5. Embo J 2005;24:1-10. [PubMed: 15577939]

27. Groves MR, Barford D. Topological characteristics of helical repeat proteins. Curr. Opin. Struct. Biol 1999;9:383-389. [PubMed: 10361086]

28. Conti E, Izaurralde E. Nucleocytoplasmic transport enters the atomic age. Curr. Opin. Cell Biol 2001;13:310-319. [PubMed: 11343901]

29. Nothwehr SF, Ha SA, Bruinsma P. Sorting of yeast membrane proteins into an endosome-to-Golgi pathway involves direct interaction of their cytosolic domains with Vps35p. J Cell Biol 2000;151:297-310. [PubMed: 11038177]

30. Peter BJ, et al. BAR domains as sensors of membrane curvature: The amphiphysin BAR structure. Science 2004;303:495-499. [PubMed: 14645856]

\section{Supplementary Material}

Refer to Web version on PubMed Central for supplementary material. 


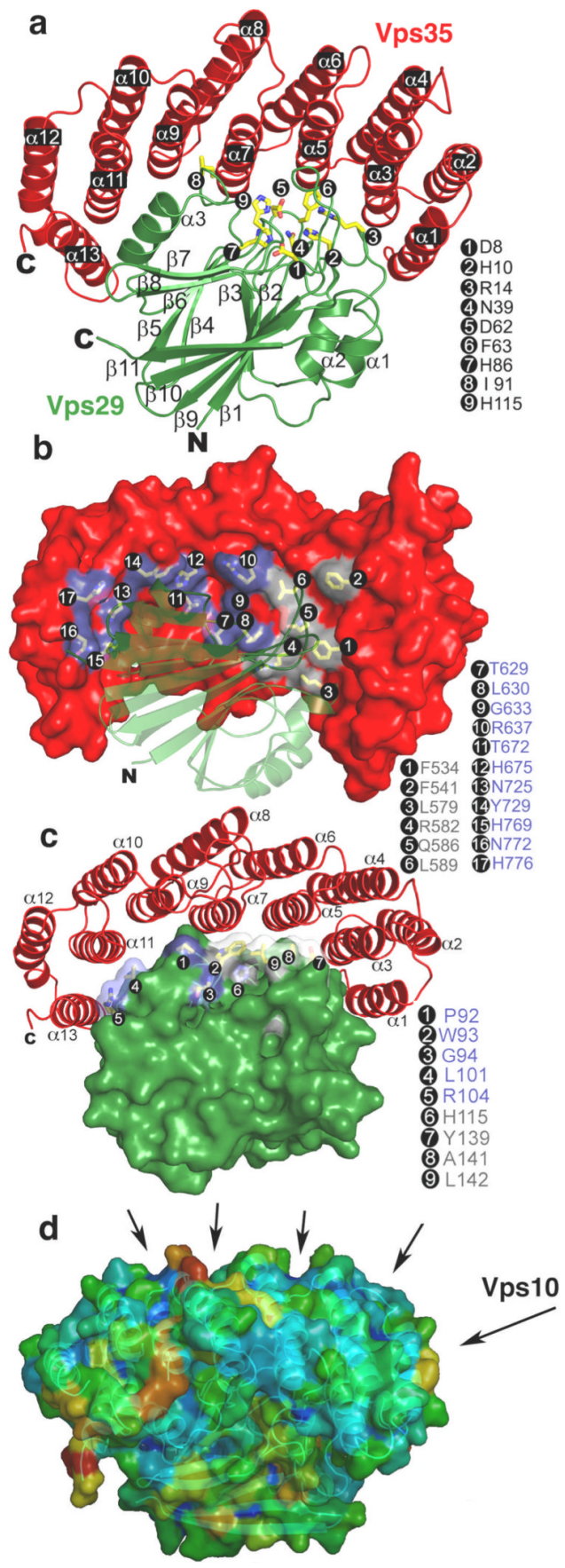

Figure 1. Structure of the Vps29:Vps35 subcomplex

a, Vps29 is green, and Vps35 red. b. The surface of Vps35, with the residues blocking the metallophosphoesterase site of Vps29 in grey, and other residues that contact Vps29 in slate. c, The surface of Vps29, with residues surrounding the metallophosphoesterase site in light blue, and other Vps35 contacting residues in slate. d, Hydrophobic grooves on the outer surface of Vps35 are formed between even-numbered helices. The probability of the surface to participate in ligand binding was colored from lowest to highest in a blue to red gradient using the hotpatch server http://hotpatch.mbi.ucla.edu/. Structural figures were generated with pymol, http://www.pymol.org/. 

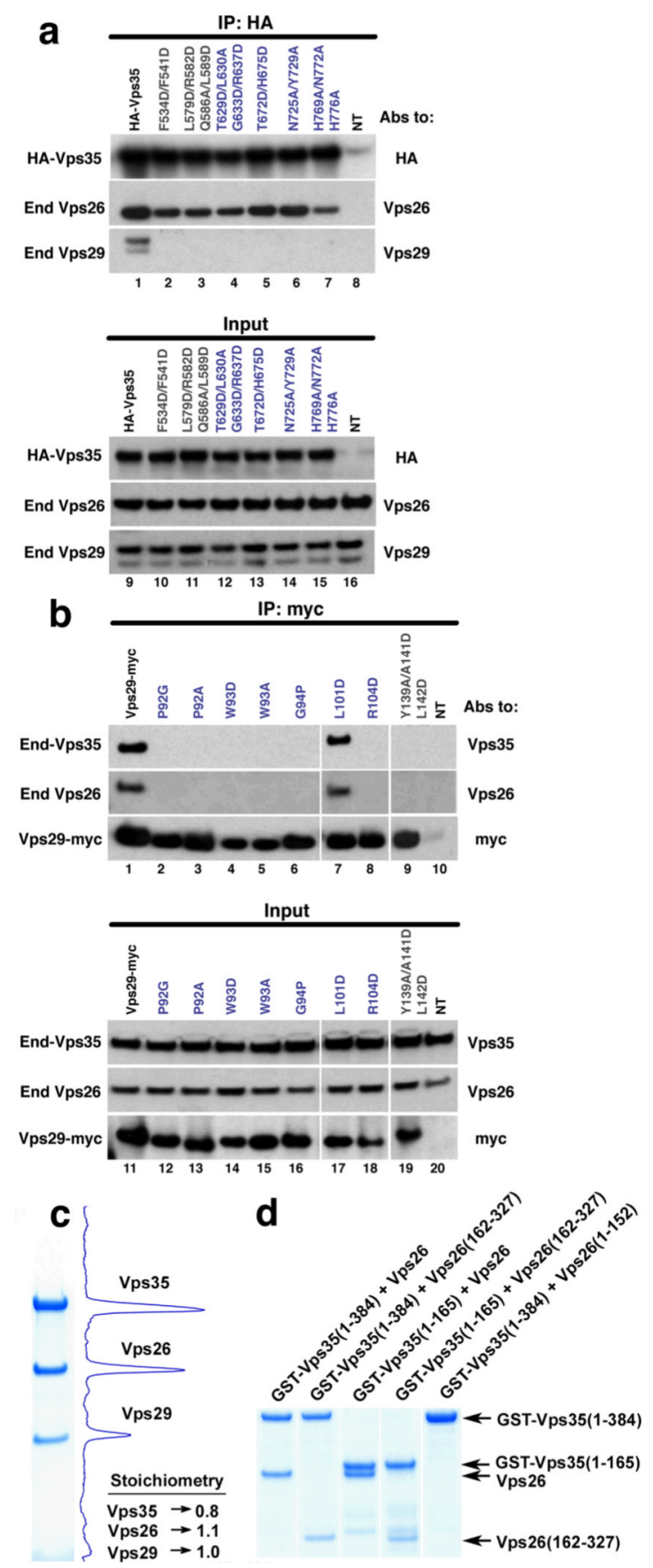

Figure 2. Assembly of the cargo recognition complex

a, Mutations in the Vps29-interaction surface of Vps35 block assembly in vivo. Lysates from HeLa cells transfected with cDNAs encoding wild-type or mutant forms of HA-tagged Vps35, or lysates from untransfected cells (lanes 8 and 16, NT) were subjected to immunoprecipitation (IP) using mouse monoclonal antibody to the HA epitope. Lysates (2\% of total, lanes 9-16) and immunoprecipitates (lanes 1-8) were analyzed by SDS-PAGE and immunoblotting with antibodies (Abs) to the HA tag, Vps26 and Vps29. b, Mutations in the Vps35-interaction surface of Vps29 abrogate assembly, with the exception of L101D. Lysates from HeLa cells transfected with cDNAs encoding wild-type or mutant Vps29-myc, or lysates from untransfected cells (lanes 10 and 20, NT) were subjected to IP using a mouse monoclonal 
antibody to the myc epitope. Lysates (5\% of total, lanes 11-20) and immunoprecipitates (lanes 1-10) were analyzed by SDS-PAGE and immunoblotting with antibodies to Vps35, Vps26 and the myc tag. c, The cargo recognition complex is an equimolar complex of Vps26, Vps29, and Vps35. Gels were stained with Coomassie blue (Simply Blue ${ }^{\mathrm{TM}}$ SafeStain; Invitrogen). Image acquisition and analysis was done in an Epichemi Darkroom (UVP BioImaging Systems) using Labworks 4.5 software. The integrated area from each peak was normalized to the calculated molecular mass for each protein, and the value was determined relative to Vps29. d, Coomassie-stained gel showing that the N-terminus of Vps35 forms a stable subcomplex with the C-terminal lobe of Vps26. For each lane, GST-tagged Vps35 fragments and untagged Vps26 or its fragments were co-expressed in E. coli and purified by glutathioneSepharose chromatography. 


\section{a}
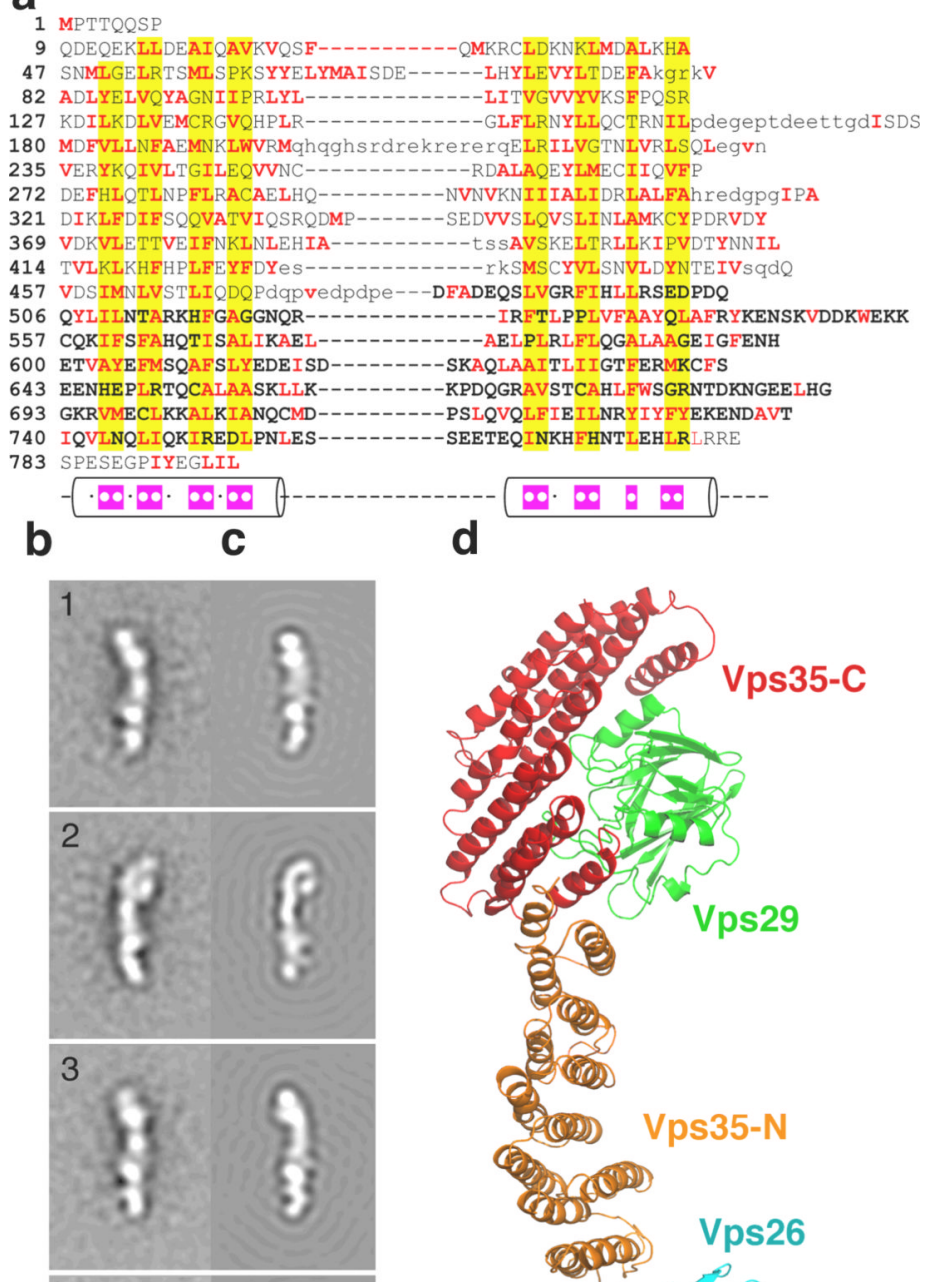

$$
\text { a }
$$
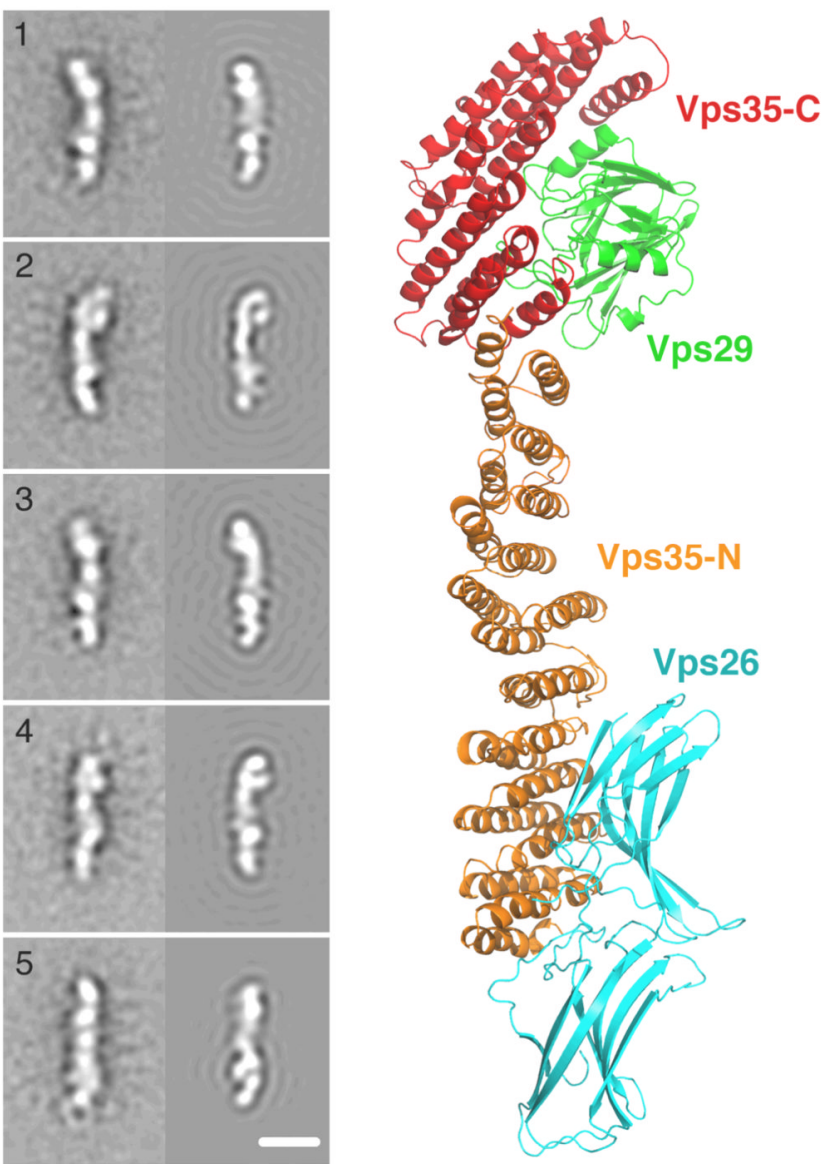

Figure 3. Structural analysis of the complete cargo recognition complex

a, Alignment of human Vps35 repeats. Columns of residues that are likely to form the hydrophobic core of the structure have yellow background. Apolar residues are in red. Cylinders show the $\alpha$-helical regions. Magenta boxes within the $\alpha$-helices indicate the positions of apolar residues in the consensus sequences. The C-terminal region of the crystal structure is in bold. Loop regions predicted based on the multiple sequence alignment of $\mathrm{Vps} 35$ proteins are shown by lower case letters. b, 1-5. Averaged images of the cargo recognition complex from negative stain electron microscopy (Fig. S6). The images were obtained by multivariate statistical analysis with reference-free alignment. The number of images per class 1 to 5 is respectively $288,362,367,206,327$. c, $1-5$. Corresponding projections, limited to $25 \AA$ 
resolution, of the cargo recognition complex model shown in $\mathbf{d}$ (C-terminal crystallized region of Vps35, red; Vps29, green; Vps26, cyan; and N-terminal modeled region of Vps35, orange). Each image in $\mathbf{c}$ is oriented such that $\mathrm{Vps} 35: \mathrm{Vps} 29$ corresponds always to the top part of the image and Vps26 to the bottom part, as in $\mathbf{d}$. The correlation coefficient between the EM class average and the corresponding model projection is for class 1 to $50.79,0.70,0.86,0.75,0.73$ respectively. Scale bar in c-5 $=100 \AA$. 

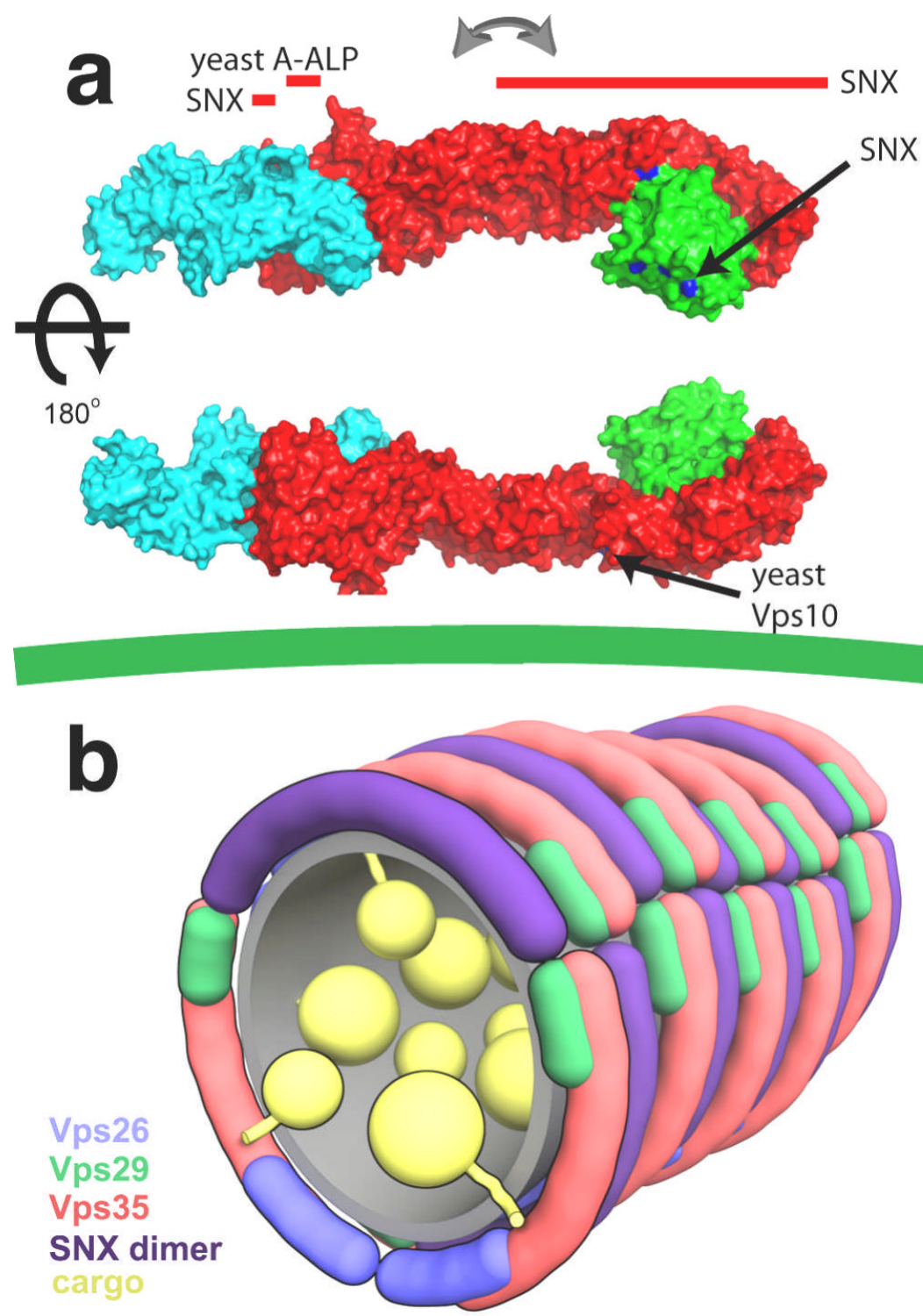

Figure 4. Integration of cargo and targeting signals by the cargo recognition complex a, The Vps26:Vps29:Vps35 complex is predicted to align roughly parallel to the membrane (green line at bottom), such that its multiple SNX 4,15 and cargo-binding sites 25 cooperatively interact. The arrows mark the central region about which $\mathrm{Vps} 35$ is proposed to flex so as to interact with cargo embedded in curved membranes. Binding sites that have been mapped to individual residues within crystallized components are shown in blue. Binding sites that have been mapped to regions of $\mathrm{Vps} 35$ or to as yet uncrystallized portions of $\mathrm{Vps} 35$ are marked by red bars aligned with the region of interest. Binding sites for yeast cargo proteins are not necessarily conserved in human Vps35, however the overall architecture of the yeast and other orthologous complexes is proposed to be very similar to the human complex. b, Schematic rendering of a speculative model for the retromer coat on a tubular vesicle, colored as above, with the SNX dimer in purple. 